\title{
Inhibition and reversal of growth cone collapse in adult sensory neurons by enteric glia-induced neurotrophic factors
}

\author{
Simon Feng ${ }^{1,2}, K_{i r a n}$ Reddy ${ }^{1,2}$, Cai-Xin Su ${ }^{1,2}$, Shu-Cui Jiang ${ }^{1,2}$ \\ ${ }^{1}$ Department of Surgery (Neurobiology), McMaster University, Health Sciences Centre, Hamilton, ON L8S 4K1, Canada. \\ ${ }^{2}$ Hamilton NeuroRestorative Group, McMaster University, Health Sciences Centre, Hamilton, ON L8S 4K1, Canada.
}

Correspondence to: Prof. Shu-Cui Jiang, Department of Surgery (Neurosurgery, Neuroscience and Neurobiology), Head of Hamilton NeuroRestorative Group, McMaster University, 1280 Main Street West HSC 4E, Hamilton, ON L8S 4K1, Canada. E-mail: jiangs@mcmaster.ca

How to cite this article: Feng S, Reddy K, Su CX, Jiang SC. Inhibition and reversal of growth cone collapse in adult sensory neurons by enteric glia-induced neurotrophic factors. Neuroimmunol Neuroinflammation 2016;3:180-8.

Article history:
Received: $05-01-2016$
Accepted: 12-07-2016
Published: $31-08-2016$
Key words:
Enteric glia
growth cone collapse
nerve growth factor
brain-derived neurotrophic factor
glial-derived neurotrophic factor
neurotrophin-3

\begin{abstract}
Aim: Previous studies show enteric glia (EG)-conditioned medium promotes neurite outgrowth in adult dorsal root ganglia (DRG) derived sensory neurons. This EG-conditioned medium contains various neurotrophic factors, including nerve growth factor (NGF), brainderived neurotrophic factor (BDNF), glial cell line-derived neurotropic factor (GDNF), and neurotrophin-3 (NT-3). This study attempts to determine the importance of these neurotrophic factors in enabling DRG-derived sensory neuron axons to overcome the inhibitory guidance cues released from the glial scar. Methods: A Semaphorin 3A (SEMA3A) growth cone collapse model was used on cultured rat DRG. Neutralizing antibodies to each neurotrophic growth factor in question (NGF, BDNF, GDNF and NT-3) were applied to the EG-conditioned medium to evaluate the factor's individual importance in preventing growth cone collapse. Results: EG-conditioned medium inhibits and reverses growth cone collapse in adult DRG neurons when added either $1 \mathrm{~h}$ before or concurrently with SEMA3A. When administered 40 min after the initial SEMA3A-induced collapse, EG-conditioned medium was able to reverse the growth cone collapse. Individual inhibition of all the neurotrophic factors, except for BDNF in the co-treatment setting, resulted in increased growth cone collapse. Conclusion: NGF, BDNF, GDNF, and NT-3 are all variably involved in preventing or reversing SEMA3Ainduced growth cone collapse in pre-, co-, and post-treatment time settings. However, no individual neurotrophic factors appear to be essential to promoting neurite outgrowth.
\end{abstract}

\section{INTRODUCTION}

Spinal cord injury $(\mathrm{SCl})$ can result in a complete or partial loss of sensation and paralysis at and below the site of injury and represents a large burden of disease. ${ }^{[1]} \mathrm{SCl}$ damage occurs in 2 phases: the primary and secondary phase. The primary phase is the membrane shearing and axonal tearing caused by mechanical stress. ${ }^{[1]}$ The secondary phase is the ischemia, apoptosis, and necrosis that occurs post-trauma. ${ }^{[2,3]}$ Glial scarring at

(i) (5) This is an open access article distributed under the terms of the Creative Commons AttributionNonCommercial-ShareAlike 3.0 License, which allows others to remix, tweak, and build upon the work non-commercially, as long as the author is credited and the new creations are licensed under the identical terms.

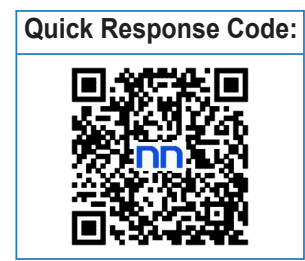


the injury site is a major mechanical barrier to axonal regeneration. Studies have found that the glial scar contains many molecules that inhibit axonal growth. ${ }^{[4-8]}$ Semaphorin 3A (SEMA3A) is one of these molecules and is highly expressed in the glial scar. ${ }^{[9-11]}$ SEMA3A is part of the Semaphorin family of inhibitory guidance cues specific to vertebrae, and it is normally present during the development of the central and peripheral nervous systems. ${ }^{[12-14]}$ Semaphorins signal through complex neuronal receptors that contain neuropilin-1 and plexins, which are significantly expressed in axotomized dorsal root ganglion (DRG). ${ }^{[13]}$ This indicates that SEMA3A is a key mediator of axon retraction and growth cone collapse. ${ }^{[13,15]}$ To date, multiple studies have observed the effects of SEMA3A on DRG-derived neurons in animal models. ${ }^{[16-21]}$ Two of them employed growth cone collapse assays adapted from studies on embryonic neurons. ${ }^{[16,18]}$

Enteric glia (EG) are the support cells of the enteric nervous system (the nervous system of the gut) and share a number of characteristics with central nervous system (CNS) astrocytes. It has been previously reported that in vivo locally transplanted EG facilitate ingrowth of transected dorsal root axons toward their targets through the spinal cord and across the nonpermissive peripheral/CNS boundary. ${ }^{[22]}$ These EG induce the regeneration of neurofilament-positive dorsal root axons into the injury site of rats given spinal cord crushes (using the clip-compression model). ${ }^{[23]}$ Additionally, there is published data that suggests EG secrete nerve growth factor (NGF), brain-derived neurotrophic factor (BDNF), glial cell line-derived neurotrophic factor (GDNF), and neurotrophin-3 (NT-3) in culture and that these growth factors at least partially mediate neurite outgrowth in DRG neurons in response to EG. ${ }^{[24]}$ Studies have shown that all of these growth factors imbue some resistance to SEMA3A-induced growth cone collapse in cultured embryonic DRG neurons, ${ }^{[25,26]}$ although collapse sensitization varies with developmental stage. ${ }^{[26-28]}$ A study by Wanigasekara et al. ${ }^{[18]}$ is of particular importance to our study, as it indicated that NGF, GDNF, and NT-3 inhibit SEM3A-induced collapse in adult DRG-derived sensory neurons when administered $1 \mathrm{~h}$ before collapse.

We hypothesized that incubation with EG-conditioned medium would inhibit and perhaps reverse SEMA3Amediated growth cone collapse in cultured DRG neurons, and theorized that inhibiting any one of the neurotrophic factors in the EG-conditioned medium would decrease its overall efficacy at preventing SEMA3A-mediated collapse, regardless of whether the conditioned medium was applied before, during, or after the addition of SEMA3A. The objective of this study was to provide evidence that neurotrophic factors are involved in mediating axonal regeneration and neurite outgrowth as well as shed light on the roles of specific neurotrophic factors in mediating neurite outgrowth.

\section{METHODS}

\section{Enteric glia extraction and culture}

All experiments were performed in accordance with the requirements of the Animals for Research Act of Ontario, Canada and the Guidelines of the Canadian Council on Animal Care and were approved by the Animal Research Ethics Board at our institution. Both EG extraction from the small intestines of adult female Wistar rats and EG identification confirmation by staining for glial fibrillary acidic protein and myelin protein zero (MPZ) were performed using previously described methods ${ }^{[29]}$ as adapted in Hansebout et al. ${ }^{[24]}$ The presence of glial fibrillary acidic protein, in conjunction with the absence of MPZ, is considered to be indicative of EG. ${ }^{[21,24]}$ EG were cultured as per ${ }^{[16]}$ in Dulbecco's modified eagle medium (DMEM/F) 12 1:1 (Invitrogen D8437) supplemented with $10 \%$ fetal bovine serum (FBS) and $1 \%$ penicillin/streptomycin $(\mathrm{P} / \mathrm{S})$. The medium was changed every 2-3 days, and the cells were then subcultured using $0.25 \%$ trypsin-ethylene diamine tetraacetic acid (Invitrogen 25200-056).

\section{DRG extraction and culture}

DRG were extracted from six- to sixteen-week old adult male Wistar rats and cultured as per Hall[ ${ }^{[30]}$ and as described by Hansebout et al., ${ }^{[24]}$ plating $3 \times 10^{4}$ cells/well in a 6 -well tissue culture plate. Non-neuronal cells were reduced with a $4 \mathrm{~h}$ incubation in dispase/ collagenase. They were then plated on a standard petri dish. Neurons generally have difficulty adhering to the petri dish surface, and the neurons mixed in solution were subsequently removed and replated. As per Reza et al., ${ }^{[16]}$ each well was coated with $3 \mathrm{~mL}$ of $0.01 \%$ poly-L-lysine $48 \mathrm{~h}$ before extraction. The polyL-lysine was replaced with $2 \mathrm{ng} / \mathrm{mL}$ laminin in HBSS $24 \mathrm{~h}$ before extraction to facilitate DRG attachment. The neurons were acclimatized to culture conditions for 4 days before each experiment. Half of the $3 \mathrm{~mL}$ of supplemented neurobasal medium was replaced with fresh medium every other day.

\section{Generation of enteric glial-conditioned medium} The EG medium was conditioned as per Hansebout et al. ${ }^{[24]}$ EG derived from adult male Wistar rats were seeded at $4 \times 10^{4}$ cells/well into a six-well plate coated with rat tail collagen, with tissue culture inserts covered in $3 \mathrm{~T} 3$ mouse embryonic fibroblasts. 
The EG were bathed in DMEM/F12 medium (Invitrogen 11330-032) supplemented with $20 \%$ FBS and $1 \%$ P/S. EG from passages 6-10 were acclimated to DMEM/ F12 containing $2 \%$ FBS and $1 \% \mathrm{P} / \mathrm{S}$. Twenty-four hours later, cells were rinsed in phosphate buffered saline (PBS, pH 7.35) and subsequently bathed in supplemented neurobasal medium. After another $24 \mathrm{~h}$, the medium was conditioned and centrifuged for $5 \mathrm{~min}$ at $4,000 \mathrm{~g}$ to remove any particulates.

\section{Collapse assay}

A collapse assay was adapted from Reza et al..[16] and experimental conditions were modified from Hansebout et al..[2] EG-conditioned medium was administered before, during or after SEMA3A (R\&D System 1250-S3) mediated collapse (pre-treatment, co-treatment, post-treatment). Each time setting was assessed using serial assays. To test individual growth factor involvement, antibodies to one of NGF, BDNF, GDNF or NT-3 were added to select wells. Each neurotrophic factor was investigated with or without conditioning media (+/-CDN) and with or without antibody (+/-aB). The negative (model; $-\mathrm{CDN} /-\mathrm{aB})$ and positive (+CDN/-aB) control groups show the effect of enteric glia on SEMA3A-mediated collapse before the addition of the antibodies. Cells were incubated at $37^{\circ} \mathrm{C}$ and PBS was used as a vector control for antibodies in each experiment arm. The final working concentration of SEMA3A in all of the wells was $100 \mathrm{ng} / \mathrm{mL}$. The final antibody concentration in each well was $2.5 \mu \mathrm{g} / \mathrm{mL}$. All cells were later fixed in $4 \%$ perfluoroalkoxy alkanes (PFA) containing $10 \%$ sucrose and subsequently stained. The detailed procedures are as follows:

\section{Pre-treatment}

Half of the medium in each well was replaced with EG-conditioned medium or normal supplemented neurobasal medium for positive and negative controls respectively. Each EG-conditioned and control neurobasal group also received either anti-NGF (RD System AMK0208091), anti-BDNF (Millipore AB15130P), anti-GDNF (RD System AFW0408071), or anti-NT-3 (Chemicon AB1780SP) dissolved in PBS to reach a working concentration of $2.5 \mu \mathrm{g} / \mathrm{mL}$ while controls were given PBS in equal volumes. After a 1-h incubation, all cultures were then treated with $100 \mu \mathrm{g} / \mathrm{mL}$ SEMA3A in PBS (R\&D Systems 1250-S3025) to obtain a bath concentration of $100 \mathrm{ng} / \mathrm{mL}$ as per Wanigasekara et al. ${ }^{[18]}$ and Reza et al. ${ }^{[16]}$ One hour later, cells were fixed and stained.

\section{Co-treatment}

This experiment was almost identical to the previous one with the exception of the timing of the treatment. Half of the medium in each well was replaced with
EG-conditioned medium or normal supplemented neurobasal medium for the comparison groups containing $200 \mathrm{ng} / \mathrm{mL}$ SEMA3A to obtain a bath concentration of $100 \mathrm{ng} / \mathrm{mL}$. The medium in the treatment groups also contained either anti-NGF, antiBDNF, anti-GDNF, anti-NT-3 added in PBS to achieve a well concentration of $2.5 \mu \mathrm{g} / \mathrm{mL}$. Equal volumes of PBS were added to control wells. The cultures were incubated for $1 \mathrm{~h}$ and then fixed and stained.

\section{Post-treatment}

Similar to the previous experiments, half of the medium in each well was replaced with fresh neurobasal medium containing $200 \mathrm{ng} / \mathrm{mL}$ SEMA3A to obtain a bath concentration of $100 \mathrm{ng} / \mathrm{mL}$. After a 40 -min incubation, $2.5 \mathrm{~mL}$ of the bathing medium was replaced with either EG-conditioned medium with SEMA3A or supplemented neurobasal medium with SEMA3A. Pilot studies revealed that $1.5 \mathrm{~mL}$ of EG medium was inadequate to prevent collapse after SEMA3A administration in our model. Also added was either anti-NGF, anti-BDNF, anti-GDNF, antiNT-3 in PBS for a final concentration of $2.5 \mathrm{ng} / \mathrm{mL}$ or equal volumes of PBS for controls. These cells were incubated for another 20 min for a total 1-h incubation period before fixing and staining.

\section{Staining}

Phalloidin staining, which specifically binds to F-actin, was used to visualize the actin-dense cytoskeleton of the growth cones. ${ }^{[31,32]}$ Specific methods were modified from Hansebout et al. ${ }^{[24]}$ After a 10-min fixation in a 4\% PFA and $10 \%$ sucrose solution, the cells were washed in PBS, permeabilized with $0.05 \%$ Triton X-100 for 5 min, and then treated with $1 \%$ bovine serum albumin for $30 \mathrm{~min}$ to reduce background staining. The cells were then stained for $1 \mathrm{~h}$ with Alexa-488 phalloidin (Invitrogen A12379) to visualize the growth cones and cell morphology and then counter-stained with Propidium iodide (Sigma) to visualize the nuclei. After a double wash in PBS and a final wash in doubledistilled water, coverslips were mounted onto glass slides using VectaShield mounting medium (Vector Laboratories $\mathrm{H}-1,000$ ), and edges of the coverslips were sealed with clear nail polish.

\section{Data gathering}

The ratio of collapsed to uncollapsed growth cones was measured as per Kapfhammer et al. ${ }^{[33]}$ and Brown et al. ${ }^{[34]}$ noting the approximate cell body size of each neurite, so as to exclude large-diameter DRG neurons that have been shown to be unresponsive to SEMA3A. ${ }^{[16]}$ Since the study used isolated DRG from adult rats rather than explanted embryonic DRG as used in the study by Kapfhammer et al. ${ }^{[33]}$ growth 
cone morphology was not as obvious; therefore, we examined the stained neurons under the $40 \times$ objective of a Leica fluorescent microscope. A minimum of 20 neurite-containing photographs were taken per slide and the first 50 different neurons were counted per slide in a horizontal strip manner. Growth cones were scored as "uncollapsed" (any flattened lamellipodia and 2 or more filopodia) or "collapsed" (bulletshaped neurite tip sometimes with a single filopodium originating at the neurite tip and no lamellipodium), as previously described. ${ }^{[16]}$ Only axons that were longer than the majority of other axons were scored; axons that were in contact with another cell surface were ignored. The results are represented as the percentage of collapsed growth cones out of the total number of growth cones counted.

\section{Statistical analysis}

Analyses were performed on combined data from at least 3 separate experiments $(n=150)$ using Graphpad 6.0, with 95\% confidence intervals. Data were plotted as the mean \pm the standard error of the mean and were compared by a two-way ANOVA followed by a Dunnett's post hoc test, comparing the means to the positive control $(+\mathrm{CDN} /-\mathrm{aB})$. Differences were significant if $P<0.05$.

\section{RESULTS}

\section{Pre-treatment}

Will inhibition of NGF, BDNF, GDNF, and NT-3 significantly decrease the effect of the EG-conditioned medium on growth cones in a SEMA3A-mediated collapse model when the conditioned medium is administered to DRG neurons prior to SEMA3A application?

In this collapse assay, DRG were incubated in either EG-conditioned medium or supplemented neurobasal medium for $1 \mathrm{~h}$. Antibodies or PBS (for controls) were also added at this time. One hour later, SEMA3A was applied. This is the first study to test the hypothesis that pre-treatment with EG-conditioned medium can prevent SEMA3A-induced growth cone collapse, as shown in Figure 1. The red bar represents the SEMA3A model (-CDN/-aB) and demonstrates that normal ranges of collapse were observed. The orange bar represents the percentage of collapse when DRG were treated with EG-conditioned medium only (+CDN/-aB). Comparing the SEMA3A model with the cultured DRG in the EG-conditioned medium demonstrates that the EG-conditioned medium (orange bar) had a positive effect on growth cones, significantly preventing their collapse in a pre-treatment setting $(P<0.0001)$. The supplemental neurobasal medium with antibody

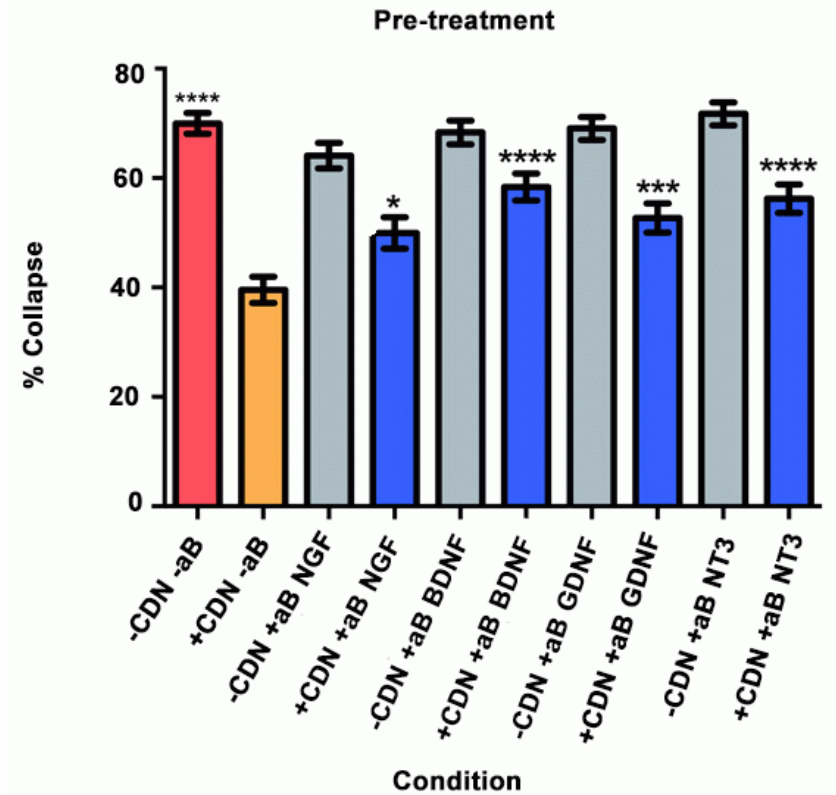

Figure 1: Pre-treatment experiment results. ${ }^{*} P<0.05$; ${ }^{* * *} P<0.001$; ${ }^{*} P<0.0001$

comparison [Figure 1, grey bars] was included to ensure that antibodies alone would not have an effect on growth cone collapse.

This experiment also tested the hypothesis that by inhibiting a specific neurotrophic factor, the ability of EG-conditioned medium to prevent SEMA3A-induced collapse would be decreased. In all of the groups treated with EG-conditioned medium and an inhibitory antibody (blue bars), the percentage of growth cone collapse was significantly higher when compared with the EG-conditioned medium alone (orange bar) (anti-NGF, $P<0.05$; anti-BDNF, $P<0.0001$; antiGDNF, $P<0.001$; anti-NT3, $P<0.0001$ ) [Figure 1]. Representative DRG neuron images from each subgroup in the pre-treatment group appear in Figure 2.

\section{Co-treatment}

Will inhibition of NGF, BDNF, GDNF, and NT-3 significantly decrease the effect of the EG-conditioned medium on growth cones in a SEMA3A-mediated collapse model when the conditioned medium is administered to DRG neurons concurrently with SEMA3A?

In this collapse assay, SEMA3A and EG-conditioned medium were added concurrently to the DRG cultures. Antibodies or PBS (for controls) were also added at this time. The supplemental neurobasal medium with antibody comparison [Figure 3, grey bars] was included to ensure that antibodies alone would not have an effect on growth cone collapse. 
The red bar represents the SEMA3A model (-CDN/$\mathrm{aB}$ ) and demonstrates that normal ranges of collapse were observed [Figure 3]. The orange bar represents the percentage of collapse when DRG were treated with EG-conditioned medium only $(+C D N /-a B)$. Comparing the SEMA3A model with the cultured DRG in the EG-conditioned medium demonstrates that the EG-conditioned medium (orange bar) had a positive effect on growth cones, significantly preventing their collapse in a co-treatment setting $(P<0.0001)$.

In the groups treated with both EG-conditioned medium and an inhibitory antibody against NGF, GDNF, and NT-3 (blue bars), the percentage of growth cone collapse was significantly greater when compared
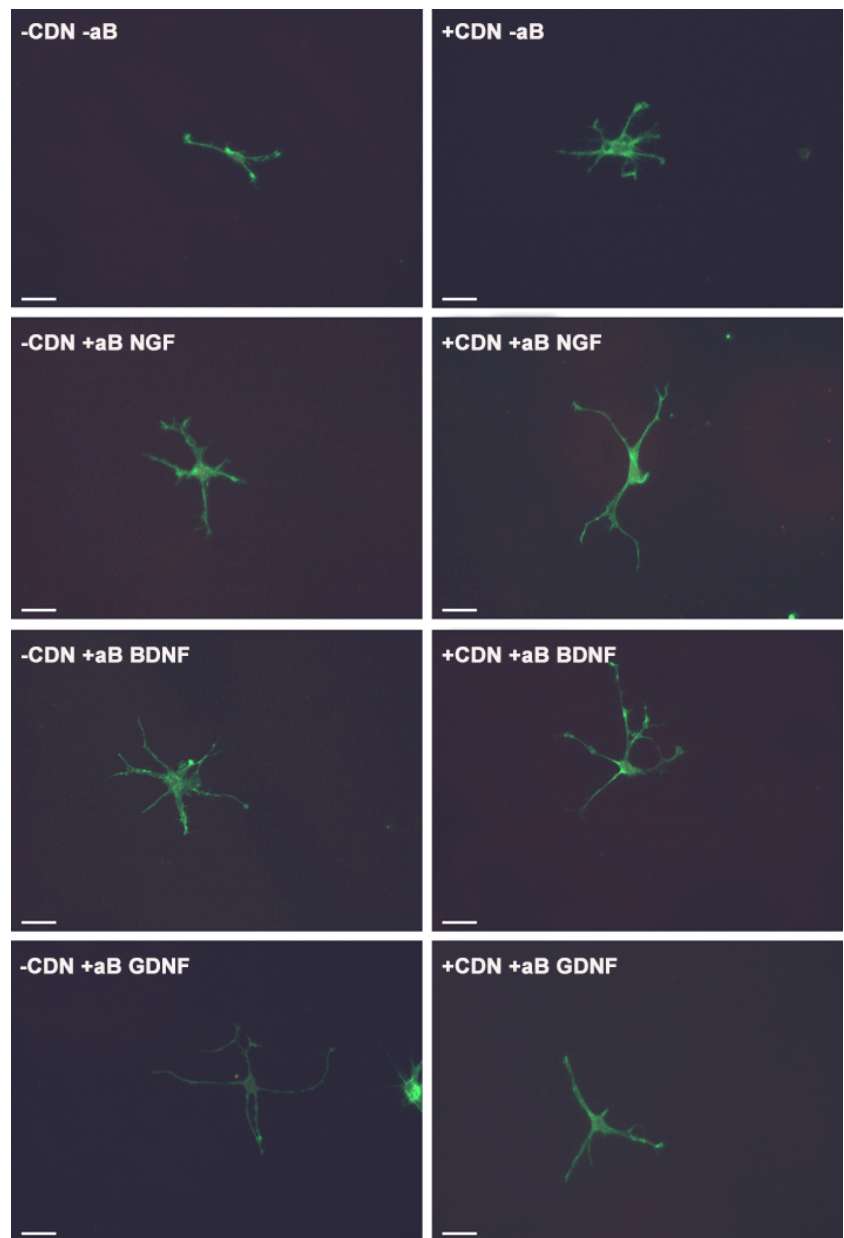

+ CDN +aB GDNF
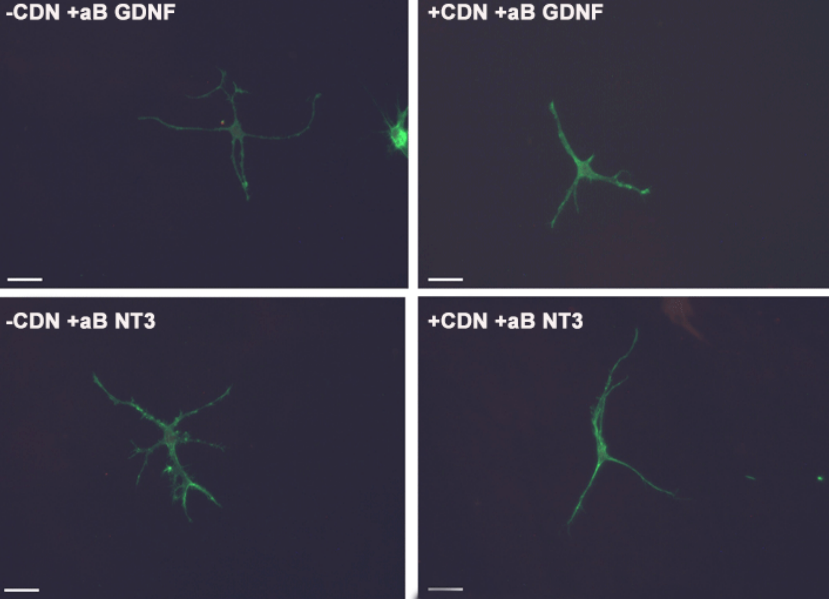

Figure 2: Images of DRG neurons from each sub-group in the pretreatment experiment. Each white bar represents $25 \mu \mathrm{m}$. DRG: dorsal root ganglion

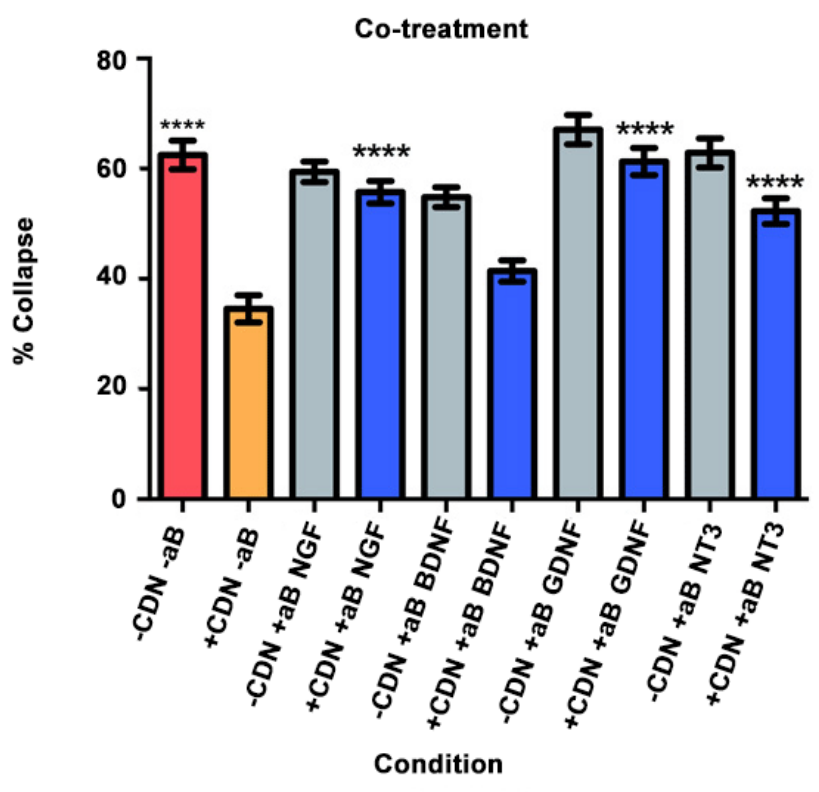

Figure 3: Co-treatment experiment results. ${ }^{* * *} P<0.0001$

with the EG-conditioned medium alone (orange bar) (anti-NGF, $P<0.0001$; anti-GDNF, $P<0.0001$; antiNT3, $P<0.0001$ ) [Figure 3]. There was no significant difference between the levels of collapse with pure EGconditioned medium (orange bar) and EG-conditioned medium with anti-BDNF antibody (blue bar; $P>0.05$ ). Representative DRG neuron images from each subgroup in the co-treatment group are shown in Figure 4.

\section{Post-treatment}

Will inhibition of NGF, BDNF, GDNF, and NT-3 significantly decrease the effect of the EG-conditioned medium on growth cones in a SEMA3A-mediated collapse model when the EG-conditioned medium is applied to DRG neurons after SEMA3A?

We used a collapse assay similar to the one in the preand co-treatment experiments. In this setting, cultured DRG neurons were bathed in $100 \mathrm{ng} / \mathrm{mL}$ of SEMA3A in supplemental neurobasal medium for $40 \mathrm{~min}$. At that time, $2.5 \mathrm{~mL}$ of the medium was exchanged with either EG-conditioned medium with SEMA3A or supplemental neurobasal medium with SEMA3A. Also added were inhibitory antibodies or PBS (for controls). The DRG were then incubated for a final $20 \mathrm{~min}$.

As illustrated in Figure 5, the red bar represents the SEMA3A model (-CDN/-aB) and indicates that normal levels of collapse were observed in the post-treatment experiment. The orange bar illustrates that posttreatment with EG-conditioned medium (+CDN/-aB) significantly prevented and/or reversed the SEMA3Ainduced growth cone collapse when compared with the SEMA3A model (-CDN/-aB; red bar, $P<0.0001)$. 


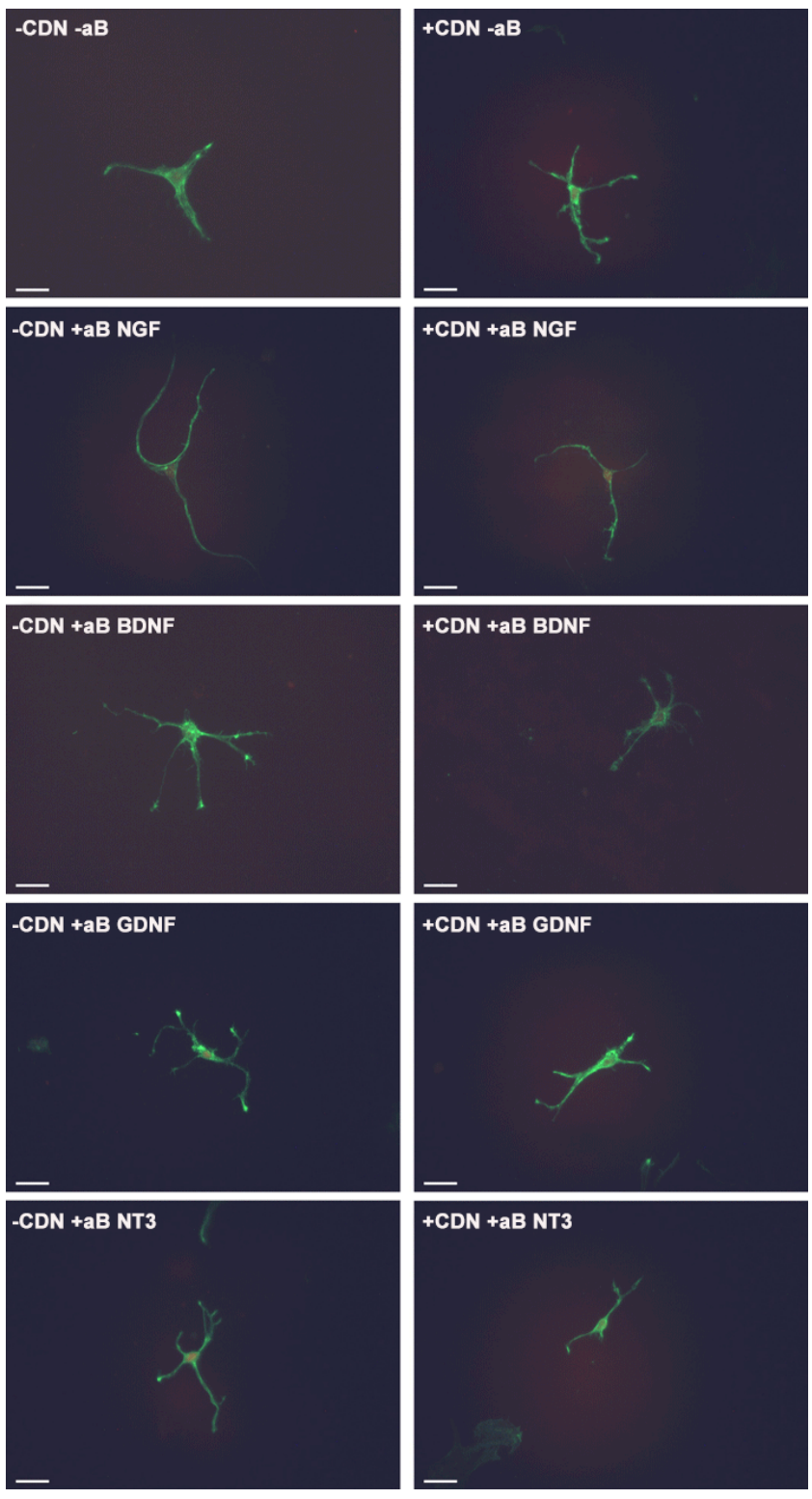

Figure 4: Images of DRG neurons from each sub-group in the cotreatment experiment. Each white bar represents $25 \mu \mathrm{m}$. DRG: dorsal root ganglion

In all groups treated with EG-conditioned medium and an inhibitory antibody (blue bars), the percentage of growth cone collapse was significantly higher when compared to EG-conditioned medium alone (orange bar) (antiNGF, $P<0.0001$; anti-BDNF, $P<0.0001$; anti-GDNF, $P<$ 0.0001; anti-NT3, $P<0.0001$ ) [Figure 5]. Representative DRG neurons images from each sub-group in the posttreatment group are shown in Figure 6.

\section{DISCUSSION}

The experiments demonstrate the novel finding that EG-conditioned medium may protect DRG growth cones against SEMA3A-mediated collapse when applied before, concurrently, and after SEMA3A.
The results show that the EG-conditioned medium, which contains NGF, BDNF, GDNF, and NT-3, is neuroprotective when applied prior to or concurrently with SEMA3A-induced collapse, and regenerative when applied 40 min after said collapse. In previous studies, it has been shown that when NGF, GDNF or neuritin is applied to DRG overnight, it is protective against SEMA3A-induced collapse. ${ }^{[18]}$ In addition, Hansebout et al. ${ }^{[24]}$ found that EG-conditioned medium expressed NGF, BDNF, GDNF, and NT-3 and could induce neurite growth in cultured DRGs.

This phenomenon may be due to either neurotrophic factor inhibition or reversal of SEMA3A-induced DRG apoptosis. ${ }^{[35]}$ Transplanted adipose derived stem cells into nerve conduits of rat DRG showed differentiation of the adipose derived stem cells and the subsequent release of NGF, BDNF, GDNF and NT-4. This was linked with a reduction in DRG mRNA expression of apoptotic factors Bax and caspase-3 and an increase in expression of the anti-apoptotic factor Bcl-2. ${ }^{[35]}$

These experiments also suggest the novel finding that NGF, BDNF, GDNF, and NT-3 are all involved in the process of preventing or reversing SEMA3Ainduced collapse, but no individual neurotrophic factor is essential to this process. In almost all cases, the application of treatment medium with inhibition of individual neurotrophic factors appeared to result in significantly increased levels of collapse compared to the pure EG-conditioned control medium. However, individual inhibition did not cause full collapse, as determined by the SEMA3A control group. This is most apparent in the pre-treatment and co-treatment

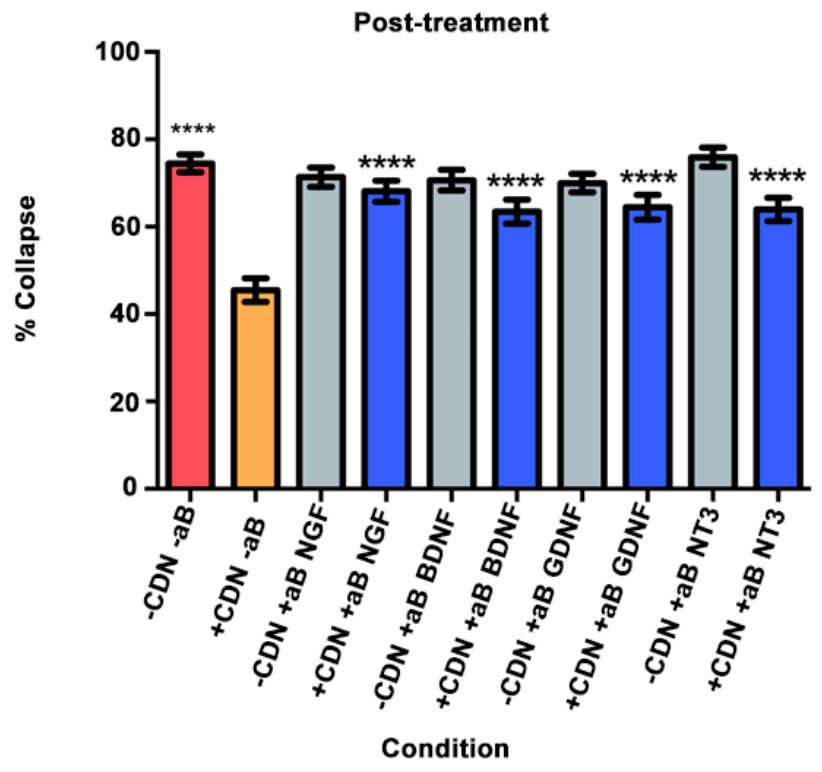

Figure 5: Post-treatment experiment results. ${ }^{* \star *} P<0.0001$ 

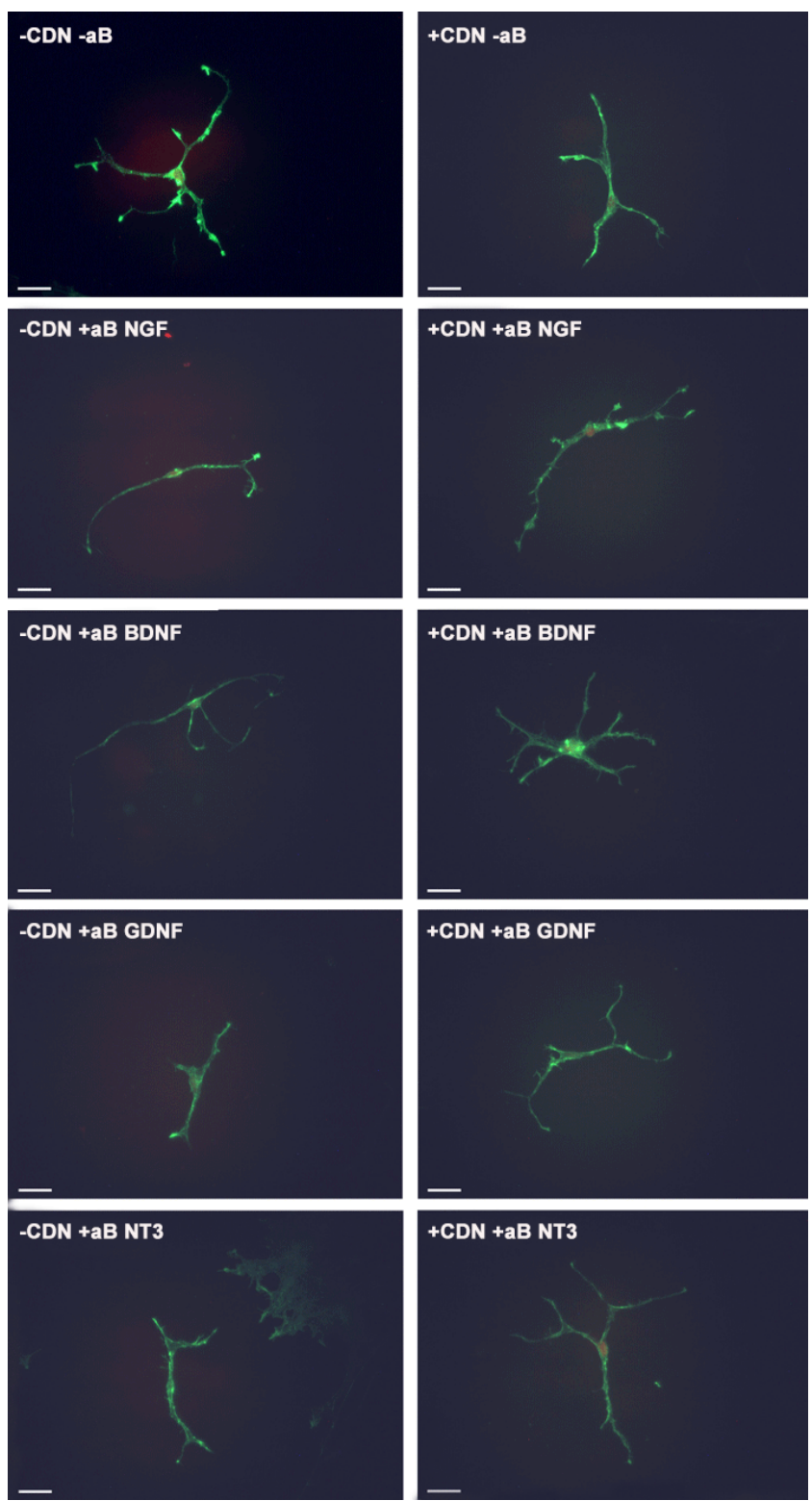

Figure 6: Images of DRG neurons from each sub-group in the posttreatment experiment. Each white bar represents $25 \mu \mathrm{m}$. DRG: dorsal root ganglion

settings. In fact, inhibition of BDNF in the co-treatment setting did not cause a significant increase in collapse, suggesting a negligible role for BDNF in that time setting. Combined, these results suggest that while these neutrophic factors play large roles, none are individually essential to the neuroprotective process.

The impact of each neurotrophic factor appears to be variable. In the pre-treatment setting, inhibition of BDNF or NT-3 resulted in the greatest increases in the percentage of growth cone collapse when compared with the pure EG-conditioned medium. This suggests that BDNF and NT-3 play the most important roles in preventing SEMA3A-induced collapse in the pretreatment setting. In the co-treatment setting, inhibition of NGF, GDNF, and NT-3 caused the greatest increases in growth cone collapse; conversely, when compared with the pure EG-conditioned medium, inhibition of BDNF did not appear to result in a significant increase in growth cone collapse. This suggests that NGF, GDNF, and NT-3 are the most important neurotrophic factors in preventing or reversing SEMA3A-induced collapse in the co-treatment setting.

In the post-treatment setting, inhibition of all neurotrophic factors resulted in significant increases in growth cone collapse when compared with the pure EG-conditioned medium. This suggests that all the neurotrophic factors in this study play an important role in reversing SEMA3A-induced growth cone collapse in a post-treatment setting. This particular setting is unique in that it tests both inhibition and reversal of growth cone collapse because it is the only time frame where SEMA3A-induced collapse had already begun before the EG-conditioned medium was introduced. This may suggest why all 4 neurotrophic factors were important in the post-treatment setting.

Previous studies have analyzed the combined effect of neurotrophic factors on neuronal development and regeneration in different settings. Madduri et al. ${ }^{[36]}$ observed that NGF and GDNF work synergistically in axon development; GDNF plays a greater role in axon elongation while NGF plays a greater role in axon branching. In another study that analyzed the length of neurite outgrowth, the results demonstrated that individual inhibition of BDNF and GDNF resulted in decreased neurite length, but inhibition of both neurotrophic factors resulted in the greatest reduction in length. ${ }^{[37]}$ Furthermore, Hansebout et al. ${ }^{[24]}$ showed that NGF, BDNF, GDNF, and NT-3 all play individual roles in DRG neurite growth. In other SEMA3A models, Wanigasekara et al. ${ }^{[18]}$ found that SEMA3A-sensitive neurons were heterogeneous in their expression of NGF, GDNF, and neuritin receptors. Their study suggests that all of these factors have a role in axonal and growth cone regeneration. Finally, Ben-Zvi et al. ${ }^{[27]}$ demonstrated that SEMA3A, NGF, BDNF, and NT-3 all play roles in determining whether DRG survive in mouse embryo models.

The post-treatment model attempts to mimic the clinical setting of post-trauma treatment of spinal cord injury. Our study is novel in that it suggests the entire complement of neurotrophins is necessary to maximally reverse and prevent further insult to the damaged area. However, no individual factor appears to be essential to the process. Since inhibition of each neurotrophic factor (NGF, BDNF, GDNF, NT-3) resulted in an increase in growth cone collapse, it suggests $E G$ 
secrete each of these factors in biologically relevant quantities in vitro. Therefore, this secretion might also be a mechanism that can mediate their beneficial effects in vivo post SCl.

Weakness of our study include that it does not verify if all neurotrophic factors were neutralized by the antibodies. While as per the manufacturer guidelines, this should have been achieved, a future study could include a secondary enzyme-linked immunosorbent assay to confirm lack of free floating neurotrophic factors. In addition, this study does not indicate if these four neurotrophic factors work together in a synergistic manner or through individual mechanisms. Similar to previous studies, further experiments where multiple neurotrophic factors are co-inhibited could shed light on this question. Furthermore, as an animal model, it has limited human application. A future study repeated in a human DRG cell line, using human EG, may provide better translational knowledge.

\section{Acknowledgments}

We thank Ms. Ann Kolkin for her critical proof reading of the manuscript and suggestions for revision.

\section{Financial support and sponsorship}

The article is supported by Canadian Spinal Research Organization \#84831.

\section{Conflicts of interest}

There are no conflicts of interest.

\section{Patient consent}

No patient involved.

\section{Ethics approval}

All experiments were performed in accordance with the requirements of the Animals for Research Act of Ontario, Canada and the Guidelines of the Canadian Council on Animal Care and were approved by the Animal Research Ethics Board at our institution.

\section{REFERENCES}

1. Dumont RJ, Okonkwo DO, Verma S, Hurlbert RJ, Boulos PT, Ellegala DB, Dumont AS. Acute spinal cord injury, part I: pathophysiologic mechanisms. Clin Neuropharmacol 2001;24:254-64.

2. Amar AP, Levy ML. Pathogenesis and pharmacological strategies for mitigating secondary damage in acute spinal cord injury. Neurosurgery 1999;44:1027-39.

3. Borgens RB, Liu-Snyder P. Understanding secondary injury. Q Rev Biol 2012;87:89-127.

4. Bolsover S, Fabes J, Anderson PN. Axonal guidance molecules and the failure of axonal regeneration in the adult mammalian spinal cord. Restor Neurol Neurosci 2008;26:117-30.

5. Chen MS, Huber AB, van der Haar ME, Frank M, Schnell L, Spillmann AA, Christ F, Schwab ME. Nogo-A is a myelin-associated neurite outgrowth inhibitor and an antigen for monoclonal antibody IN-1. Nature 2000;403:434-9.

6. McKerracher L, David S, Jackson DL, Kottis V, Dunn RJ, Braun PE. Identification of myelin-associated glycoprotein as a major myelinderived inhibitor of neurite growth. Neuron 1994;13:805-11.

7. Fawcett JW, Schwab ME, Montani L, Brazda N, Müller HW. Defeating inhibition of regeneration by scar and myelin components. Handb Clin Neurol 2012;109:503-22.

8. Hagg T, Oudega M. Degenerative and spontaneous regenerative processes after spinal cord injury. J Neurotrauma 2006;23:264-80.

9. Kaneko S, Iwanami A, Nakamura M, Kishino A, Kikuchi K, Shibata S, Okano HJ, Ikegami T, Moriya A, Konishi O, Nakayama C, Kumagai K, Kimura T, Sato Y, Goshima Y, Taniguchi M, Ito M, He Z, Toyama Y, Okano H. A selective Sema3A inhibitor enhances regenerative responses and functional recovery of the injured spinal cord. Nat Med 2006;12:1380-9.

10. Niclou SP, Ehlert EME, Verhaagen J. Chemorepellent axon guidance molecules in spinal cord injury. J Neurotrauma 2006;23:409-21.

11. Pasterkamp RJ, Giger RJ, Ruitenberg MJ, Holtmaat AJ, De Wit J, De Winter F, Verhaagen J. Expression of the gene encoding the chemorepellent semaphorin III is induced in the fibroblast component of neural scar tissue formed following injuries of adult but not neonatal CNS. Mol Cell Neurosci 1999;13:143-66.

12. Behar O, Golden JA, Mashimo H, Schoen FJ, Fishman MC. Semaphorin III is needed for normal patterning and growth of nerves, bones and heart. Nature 1996;383:525-8.

13. Pasterkamp RJ, Verhaagen J. Semaphorins in axon regeneration: developmental guidance molecules gone wrong? Philos Trans $R$ Soc Lond B Biol Sci 2006;361:1499-511.

14. Taniguchi M, Yuasa S, Fujisawa H, Naruse I, Saga S, Mishina M, Yagi T. Disruption of semaphorin III/D gene causes severe abnormality in peripheral nerve projection. Neuron 1997;19:519-30.

15. Jiang SX, Whitehead S, Aylsworth A, Slinn J, Zurakowski B, Chan K, Li J, Hou ST. Neuropilin 1 directly interacts with Fer kinase to mediate semaphorin 3A-induced death of cortical neurons. J Biol Chem 2010;285:9908-18

16. Reza JN, Gavazzi I, Cohen J. Neuropilin-1 is expressed on adult mammalian dorsal root ganglion neurons and mediates semaphorin3a/ collapsin-1-induced growth cone collapse by small diameter sensory afferents. Mol Cell Neurosci 1999;14:317-26.

17. Tang X-Q, Tanelian DL, Smith GM. Semaphorin3A inhibits nerve growth factor-induced sprouting of nociceptive afferents in adult rat spinal cord. J Neurosci 2004;24:819-27.

18. Wanigasekara Y, Keast JR. Nerve growth factor, glial cell line-derived neurotrophic factor and neurturin prevent semaphorin 3A-mediated growth cone collapse in adult sensory neurons. Neuroscience 2006;142:369-79.

19. Treinys R, Kaselis A, Jover E, Bagnard D, Šatkauskas S. R-type calcium channels are crucial for semaphorin $3 \mathrm{~A}$-induced DRG axon growth cone collapse. PLoS One 2014;9:e102357.

20. Kaselis A, Treinys R, Vosyliute R, Šatkauskas S. DRG axon elongation and growth cone collapse rate induced by Sema3A are differently dependent on NGF concentration. Cell Mol Neurobiol 2014;34:289-96.

21. Yamazaki M, Yamauchi Y, Goshima Y, Kanaho Y. Phosphatidylinositol 4-phosphate 5-kinase $\beta$ regulates growth cone morphology and semaphorin 3A-triggered growth cone collapse in mouse dorsal root ganglion neurons. Neurosci Lett 2013;547:59-64.

22. Jiang S, Wang J, Khan MI, Middlemiss PJ, Salgado-Ceballos H, Werstiuk ES, Wickson R, Rathbone MP. Enteric glia promote regeneration of transected dorsal root axons into spinal cord of adult rats. Exp Neurol 2003;181:79-83.

23. Khan M, Bain J, Hansebout C, Rathbone M. Locally transplanted enteric glia improve functional and structural recovery in a rat model of 
spinal cord injury. Neural Regen Res 2009;9:016.

24. Hansebout CR, Su CX, Reddy K, Zhang D, Jiang C, Rathbone MP, Jiang S. Enteric glia mediate neuronal outgrowth through release of neurotrophic factors. Neural Regen Res 2012;7:2165-75.

25. Dontchev VD, Letourneau PC. Nerve growth factor and semaphorin 3A signaling pathways interact in regulating sensory neuronal growth cone motility. J Neurosci 2002;22:6659-69.

26. Tuttle R, O'Leary DD. Neurotrophins rapidly modulate growth cone response to the axon guidance molecule, collapsin-1. Mol Cell Neurosci 1998;11:1-8

27. Ben-Zvi A, Yagil Z, Hagalili Y, Klein H, Lerman O, Behar O. Semaphorin $3 \mathrm{~A}$ and neurotrophins: A balance between apoptosis and survival signaling in embryonic DRG neurons. J Neurochem 2006;96:585-97.

28. Pond A, Roche FK, Letourneau PC. Temporal regulation of neuropilin-1 expression and sensitivity to semaphorin $3 \mathrm{~A}$ in NGF- and NT3responsive chick sensory neurons. J Neurobiol 2002;51:43-53.

29. Middlemiss PJ, Jiang S, Wang J, Rathbone MP. A method for purifying enteric glia from rat myenteric plexus. In Vitro Cell Dev Biol Anim 2002;38:188-90.

30. Hall AK. Rodent sensory neuron culture and analysis. Curr Protoc
Neurosci 2006; doi: 10.1002/0471142301.ns0319s36

31. Small JV, Rottner K, Hahne P, Anderson KI. Visualising the actin cytoskeleton. Microsc Res Tech 1999;47:3-17.

32. Mitchison TJ, Cramer LP. Actin-based cell motility and cell locomotion Cell 1996;84:371-9.

33. Kapfhammer JP, Xu H, Raper JA. The detection and quantification of growth cone collapsing activities. Nat Protoc 2007;2:2005-11.

34. Brown JA, Wysolmerski RB, Bridgman PC. Dorsal root ganglion neurons react to semaphorin $3 \mathrm{~A}$ application through a biphasic response that requires multiple myosin II isoforms. Mol Biol Cell 2009;20:1167-79.

35. Reid AJ, Sun M, Wiberg M, Downes S, Terenghi G, Kingham PJ. Nerve repair with adipose-derived stem cells protects dorsal root ganglia neurons from apoptosis. Neuroscience 2011;199:515-22.

36. Madduri S, Papaloïzos M, Gander B. Synergistic effect of GDNF and NGF on axonal branching and elongation in vitro. Neurosci Res 2009;65:88-97.

37. Gu W, Zhang F, Xue Q, Ma Z, Lu P, Yu B. Bone mesenchymal stromal cells stimulate neurite outgrowth of spinal neurons by secreting neurotrophic factors. Neurol Res 2012;34:172-80. 